\title{
Therapeutic Effect of Intrastromal Voriconazole, Topical Voriconazole, and Topical Natamycin on Fusarium Keratitis in Rabbit
}

\author{
Mahmood Nejabat, ${ }^{1}$ Nafiseh Yaqubi, ${ }^{2}$ Amir Khosravi, ${ }^{3}$ Kamiar Zomorodian, ${ }^{4}$ \\ Mohammad Javad Ashraf, ${ }^{5}$ and Ramin Salouti ${ }^{1}$ \\ ${ }^{1}$ Poostchi Eye Research Center, Department of Ophthalmology, Shiraz University of Medical Sciences, Shiraz 7134845794, Iran \\ ${ }^{2}$ Department of Ophthalmology, Shiraz University of Medical Sciences, Shiraz 7134845794, Iran \\ ${ }^{3}$ Yasuj University of Medical Sciences, Yasuj 7591741417, Iran \\ ${ }^{4}$ Basic Sciences in Infectious Diseases Research Center and Department of Medical Mycology and Parasitology, \\ School of Medicine, Shiraz University of Medical Sciences, Shiraz 7134845794, Iran \\ ${ }^{5}$ Department of Pathology, Shiraz University of Medical Sciences, Shiraz 7134845794, Iran
}

Correspondence should be addressed to Amir Khosravi; amirl1khosravi@gmail.com

Received 29 January 2016; Revised 27 March 2016; Accepted 18 April 2016

Academic Editor: Ilona Dóczi

\begin{abstract}
Copyright (c) 2016 Mahmood Nejabat et al. This is an open access article distributed under the Creative Commons Attribution License, which permits unrestricted use, distribution, and reproduction in any medium, provided the original work is properly cited.

Purpose. Evaluating the therapeutic effect of topical and intrastromal voriconazole and topical natamycin on Fusarium keratitis. Methods. 24 rabbits were selected. The stroma of their corneas was inoculated with suspension of Fusarium solani species complex. Seven days after injection they were divided into 4 groups randomly: the first group was treated with topical voriconazole (TV) $1 \%$ for one week, the second one with one-time intrastromal injection of voriconazole (ISV) 50 microgram/0.1 mL, and the third group with topical gel of natamycin (TN) for one week, and the last one did not receive any antifungal treatment. Finally the eyes were enucleated and sclerocorneal buttons were sent for histological and microbiological examinations. Results. After treatment the ISV group and TN group showed significantly lower clinical score and colony forming units than the control group $(P=0.040$ and $P=0.026$, resp.), but there was statistically no significant difference between control and TV groups $(P=0.249)$ or between ISV and TN groups $(P=0.665)$. In pathological evaluation, fewer chronic inflammations were reported in 2 of the 3 buttons from TV group and 3 of the 3 buttons from ISV and TN groups in comparison with the control group. Conclusion. Intrastromal injection of voriconazole seems to be effective in treatment of Fusarium keratitis as much as topical natamycin and these are more effective than topical voriconazole.
\end{abstract}

\section{Introduction}

Infectious keratitis is one of the important causes of corneal blindness which is a major public health problem worldwide [1]. The incidence of fungal keratitis varies around the world and is more prevalent in areas with hot humid climates. Many species of fungi exist as part of the normal ocular surface microbiota [2]. However, under some circumstances such as corticosteroid therapy and trauma to the eyeball, these fungi might invade the eye and cause fungal ocular infections [1].
Among these fungi, Fusarium species are the most frequent cause of fungal keratitis and account for up to one-third of these infections. They are fast-growing hyalohyphomycetes that have been isolated from soil, plants, and water [3]. Most incidences of Fusarium keratitis are caused by an eye injury with vegetative matter, such as trauma to the eye with a palm branch [4]. Fusarium keratitis infections through contact lens wear have been reported, but they are less prevalent [5].

Fusarium solani species complex (FSSC) can cause severe types of fungal keratitis because of its high level of virulence and its resistance to antifungal medications [6]. Keratitis 
caused by FSSC may lead to serious complications such as endophthalmitis, descemetocele, perforation, and blindness [7].

Considering its difficult diagnosis, fungal keratitis is one of the most serious and hazardous forms of corneal infections. Moreover, antifungal drugs are not as forceful and effective as antibacterial agents. Antifungal drugs have little corneal penetration and low efficiency [8].

Different classes of antifungals including polyenes, triazoles, and echinocandins have been used previously in the treatment of fungal keratitis [9]. In India, natamycin is used frequently as the mainstay of treatment [10]. Secondgeneration triazoles such as posaconazole and voriconazole seem to be effective in the treatment of ocular or corneal fungal infections [11].

Few studies are available about the efficacy of these drugs on fungal keratitis and the route of prescription. One of the recently suggested routes of prescription is intrastromal injection of voriconazole. Siatiri et al. [12] reported results of intrastromal voriconazole injection in the treatment of two patients with recalcitrant Fusarium keratitis. They concluded that intrastromal injection of voriconazole together with topical voriconazole effectively reduced the infiltration size and controlled the infection in patients with Fusarium keratitis.

In this study, we aimed to evaluate the therapeutic effect of intrastromal voriconazole on Fusarium keratitis and compare it with topical voriconazole and topical natamycin.

\section{Materials and Methods}

This study was performed at the Department of Ophthalmology at Khalili Eye Hospital (Shiraz University of Medical Sciences, Shiraz, Iran) and was approved by ethics committee of Shiraz University of Medical Sciences.

FSSC was isolated from a patient with fungal keratitis. The isolate was subcultured on potato dextrose agar (Merck, Germany) plates at $28^{\circ} \mathrm{C}$ to induce sporulation. The conidia were harvested by washing the surface of the colonies with $0.1 \%$ Tween 80 in the sterile physiological saline and filtering the suspension through 2 layers of sterile gauze to remove hyphal residue. The spore suspensions were then transferred to Eppendorf tubes and centrifuged at $10000 \times \mathrm{g}$ to pellet the conidia. The supernatant was carefully removed, and the conidia were resuspended with sterile normal saline to yield the final inoculum of $8.6 \times 10^{4}$ colony forming units (CFU) per milliliter. The number of conidia was measured by hemocytometer and their viabilities were determined and confirmed by quantitative plating of serial dilutions of stock inoculum.

Twenty-four New Zealand white rabbits weighing 2.5 to $3 \mathrm{~kg}$ were selected from the animal laboratory of Shiraz University of Medical Sciences. The study was restricted to rabbits that did not have any kind of keratitis. Rabbits with any kind of corneal opacity were excluded from our study. They were systemically anesthetized with $50 \mathrm{mg} / \mathrm{kg}$ of intramuscular ketamine hydrochloride and $5 \mathrm{mg} / \mathrm{kg}$ of xylazine before all interventions. The stromas of their right corneas were inoculated with $0.1 \mathrm{~mL}$ suspension of FSSC

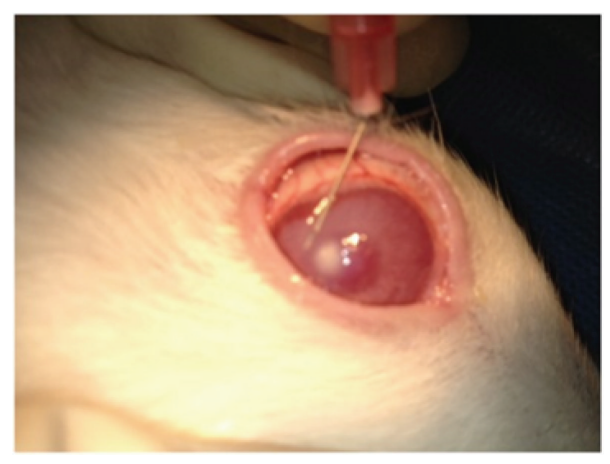

FIGURE 1: Intrastromal injection of voriconazole 50 microgram/ $0.1 \mathrm{~mL}$ in the operating room.

$\left(10^{3} \mathrm{CFU} / \mathrm{mL}\right.$ under sterile condition in the animal operating room).

One week after injection they were checked for fungal keratitis and the size of corneal ulcer and corneal clouding were measured. The rabbits were randomly divided into four groups: the first group received topical voriconazole $1 \%$ once each hour (Q1h) (except from 1 a.m. to 7 a.m.) for one week, the second one received a single injection of intrastromal voriconazole 50 microgram/0.1 mL (Figure 1), the third group received topical gel of natamycin Q1h (except from 1 a.m. to 7 a.m.) for one week, and the last group did not receive any antifungal treatment and was considered as the control group. All groups received chloramphenicol drops four times a day for 2 weeks for prophylaxis of bacterial superinfection.

The eyes in four groups were examined at day 7 (baseline) and at day 14 (7 days after beginning of treatment, before enucleation) using an operative biomicroscope.

The extent of keratitis was evaluated by a masked observer. The severity of keratomycosis in the rabbits was scored with the scoring system described by Wu et al. [13]. Each of the following criteria was graded: area of corneal opacity, density of opacity, and surface regularity (Table 1). A normal cornea was given a score of 0 in each category and a summation score of 0 . The scores from these categories were tallied for each cornea to show a possible total score ranging from 0 to 12 . A total score of 5 or less was considered mild, a total score of 6 to 9 was categorized as moderate, and a total score of more than 9 was considered severe.

On day 14 the eyes were enucleated and sclerocorneal buttons were sent for microbiological and histological examinations. In each of the examined groups, the corneal buttons were weighed, homogenized, and inoculated onto the Sabouraud dextrose agar plates. Following 2-7 days of incubation at $28^{\circ} \mathrm{C}$, CFUs/g were determined and compared.

The specimens were immediately inoculated onto the Sabouraud dextrose agar plates and incubated at $28^{\circ} \mathrm{C}$ for 7 days. The quantity of colonies in each group was determined and compared.

The corneal buttons were fixed in $10 \%$ buffered formalin for 24 hours. Then, the buttons were halved and underwent routine tissue processing and tissue block preparation. $5 \mu \mathrm{m}$ 
TABLE 1: Quantitative scoring system for rabbit Fusarium keratitis.

\begin{tabular}{|c|c|c|c|c|}
\hline & Grade I & Grade II & Grade III & Grade IV \\
\hline $\begin{array}{l}\text { Area of corneal } \\
\text { opacity }\end{array}$ & $1 \%-25 \%$ & $26 \%-50 \%$ & $51 \%-75 \%$ & $76 \%-100 \%$ \\
\hline $\begin{array}{l}\text { Density of } \\
\text { corneal opacity }\end{array}$ & $\begin{array}{c}\text { Slight } \\
\text { cloudiness, } \\
\text { outline of iris } \\
\text { and pupil being } \\
\text { discernable }\end{array}$ & $\begin{array}{l}\text { Cloudy, but } \\
\text { outline of iris } \\
\text { and pupil } \\
\text { remains visible }\end{array}$ & $\begin{array}{l}\text { Cloudy, opacity } \\
\text { not uniform }\end{array}$ & Uniform opacity \\
\hline $\begin{array}{l}\text { Surface } \\
\text { regularity }\end{array}$ & $\begin{array}{l}\text { Slight surface } \\
\text { irregularity }\end{array}$ & $\begin{array}{l}\text { Rough surface, } \\
\text { some swelling }\end{array}$ & $\begin{array}{l}\text { Significant } \\
\text { swelling, crater } \\
\text { or serious } \\
\text { descemetocele } \\
\text { formation }\end{array}$ & $\begin{array}{l}\text { Perforation or } \\
\text { descemetocele }\end{array}$ \\
\hline
\end{tabular}

TABLE 2: Mean clinical scores obtained from each group on day 7.

\begin{tabular}{lccccrr}
\hline Group & Factor & $N$ & Minimum & Maximum & Mean & \multicolumn{1}{c}{ SD } \\
\hline C & & 6 & 6.00 & 8.00 & 7.1667 & 0.75277 \\
TV & Clinical score on day 7 & 6 & 6.00 & 8.00 & 7.1667 & 0.98319 \\
ISV & & 6 & 6.00 & 8.00 & 7.1667 & 0.75277 \\
N & & 6 & 6.00 & 8.00 & 7.0000 & 0.89443 \\
\hline
\end{tabular}

C: control group, TV: topical voriconazole, ISV: intrastromal voriconazole, N: natamycin, SD: standard deviation.

TABLE 3: Mean clinical scores obtained from each group on day 14.

\begin{tabular}{lcccccc}
\hline Group & Factor & $N$ & Minimum & Maximum & Mean & Std. deviation \\
\hline C & & 6 & 4.00 & 10.00 & 6.8333 & 2.22860 \\
TV & Clinical score on day 14 & 6 & 4.00 & 10.00 & 5.5000 & 2.25832 \\
ISV & & 6 & 3.00 & 5.00 & 4.3333 & 0.81650 \\
N & & 6 & 3.00 & 5.00 & 4.1667 & 0.75277 \\
\hline
\end{tabular}

C: control group, TV: topical voriconazole, ISV: intrastromal voriconazole, N: natamycin.

thick tissue sections were prepared and stained with hematoxylin and eosin.

The sections were examined under light microscope (Olympus BX41). Presence or absence of ulceration, fungal hyphae, type and severity of inflammation, extent of stromal scarring and vascularization, and characteristics of infiltrating cells were evaluated.

The data were presented as mean $\pm \mathrm{SD}$. The statistical analyses of differences between different groups were performed using the Tukey HSD and Mann-Whitney tests. The Wilcoxon test was used to compare the results for each group between days 7 and 14. $P<0.05$ was considered as significant. All statistical analyses were done using SPSS software, version 12.

\section{Results}

Fusarium corneal ulcer was developed in all eyes one week after intrastromal injection of fungal suspension. Tables 2 and 3 show the mean clinical scores obtained from each group on days 7 and 14 .

Kruskal-Wallis one-way analysis of variance showed that statistically there was no significant difference between these four groups at baseline. Mann-Whitney $U$ test showed that,
TABLE 4: Comparisons between the four groups in terms of clinical score on day 14 .

\begin{tabular}{lcc}
\hline Groups & Mann-Whitney $U$ & Significance \\
\hline C-TV & 11.000 & 0.249 \\
C-ISV & 5.5 & 0.040 \\
C-N & 4.5 & 0.026 \\
TV-ISV & 12.5 & 0.337 \\
TV-N & 10 & 0.167 \\
ISV-N & 15.5 & 0.665 \\
\hline
\end{tabular}

C: control group, TV: topical voriconazole, ISV: intrastromal voriconazole, $\mathrm{N}$ : natamycin.

on day 14, the difference between the control group and intrastromal voriconazole group was significant statistically. The difference between control group and natamycin group was also significant, but there was statistically no significant difference between the control and the topical voriconazole group or between the intrastromal voriconazole and natamycin groups (Table 4). These data indicate that rabbits in the intrastromal voriconazole and natamycin groups developed infection with less severity than the control and topical voriconazole groups. 
TABLE 5: The values of the colony forming units obtained from the cultured samples.

\begin{tabular}{lcccccc}
\hline Group & Factor & $N$ & Minimum & Maximum & Mean & SD \\
\hline C & & 6 & 48.0 & 52.0 & 50.333 & 1.8619 \\
TV & Colony forming unit/g & 6 & 0.00 & 51.0 & 24.667 & 27.0382 \\
ISV & & 6 & 0.00 & 48.0 & 8.000 & 19.5959 \\
N & & 6 & 0.00 & 0.00 & 0.0000 & 0.00000 \\
\hline
\end{tabular}

C: control group, TV: topical voriconazole, ISV: intrastromal voriconazole, N: natamycin, SD: standard deviation.

TABLE 6: Tukey's HSD test analysis of colony forming units.

\begin{tabular}{lcccccc}
\hline Group I & Group J & Mean difference (I - J) & SE & Significance & \multicolumn{2}{c}{$\begin{array}{c}\text { 95\% confidence interval } \\
\text { Lower bound }\end{array}$} \\
\hline \multirow{2}{*}{ Control } & ISV & 42.3333 & 9.6546 & 0.002 & 15.311 & 69.356 \\
& $\mathrm{~N}$ & 50.3333 & 9.6546 & 0.000 & 23.311 & 77.356 \\
\hline
\end{tabular}

ISV: intrastromal voriconazole, $\mathrm{N}$ : natamycin, $\mathrm{SE}$ : standard error.

TABLE 7: The pathological comparison of 9 corneal buttons of the experimental groups with the control group ( 3 corneal buttons from each experimental group).

\begin{tabular}{llll}
\hline & TV & ISV & N \\
\hline Chronic inflammation & Similar to C & Fewer than C & Fewer than C \\
Eosinophils & Similar to C & Fewer than C & Fewer than C \\
Anterior stromal vascularization & Similar to C & Fewer than C & Fewer than C \\
Chronic inflammation & Fewer than C & Fewer than C & Fewer than C \\
Eosinophils & Fewer than C & Fewer than C & Fewer than C \\
Anterior stromal vascularization & Fewer than C & Fewer than C & Fewer than C \\
Chronic inflammation & Fewer than C & Similar to C & Fewer than C \\
Eosinophils & Similar to C & Similar to C & Fewer than C \\
Anterior stromal vascularization & Similar to C & Fewer than C \\
\hline
\end{tabular}

C: control group, TV: topical voriconazole, ISV: intrastromal voriconazole, $\mathrm{N}$ : natamycin.

The mean \pm SD weight of the samples sent to the microbiological laboratory was $0.1494 \mathrm{gr} \pm 0.0085$. The values about the colony forming units (CFUs) that were obtained from the cultured samples are given in Table 5 .

Tukey's HSD test showed a statistically significant difference between the control and intrastromal voriconazole groups (Table 6). There was a significant difference between the control and natamycin groups, but the differences between the control and topical voriconazole and between intrastromal voriconazole and natamycin groups were not significant statistically. These data indicate that Fusarium proliferation was significantly lower in intrastromal voriconazole and natamycin groups.

In pathological evaluation of the control group similar findings including moderate chronic inflammation, moderate eosinophils, and anterior stromal vascularization were found. The pathological comparison of the experimental groups with the control group is shown in Table 7. Plasma cells, lymphocytes, neutrophils, eosinophils, and mast cells were the inflammatory cells that were investigated.

In pathological evaluation, fewer chronic inflammations were reported in 2 of the 3 buttons from the topical voriconazole group and 3 of the 3 buttons from the intrastromal voriconazole and natamycin groups in comparison with the control group. In 1 of the 3 buttons from the topical voriconazole group, 2 of the 3 buttons from the intrastromal voriconazole group, and 3 of the 3 ones from the natamycin group, fewer eosinophils and anterior stromal vascularization were reported.

\section{Discussion}

Fungal keratitis is an important cause of ocular morbidity and blindness. The diagnosis of these infections is very difficult and currently the therapy for fungal diseases is not as forceful and effective as antibacterials. Antifungal drugs have little corneal penetration and low efficiency [7]. Alexandrakis et al. [14] reported that Fusarium spp. were the most common isolates in progressive keratitis. Lin et al. [15] reported that almost $70 \%$ of patients with deep lesions of Fusarium keratitis do not respond to medical therapy alone. FSSC is very virulent and can destroy an eye completely within a few weeks because the infection is usually severe and perforation, deep extension, and malignant glaucoma may supervene. The most commonly used topical medications for Fusarium keratitis are azole derivatives and natamycin.

Prajna et al. [16] found no difference in three-month best spectacle corrected visual acuity or scar size between 
natamycin- and voriconazole-treated patients in Fusarium keratitis. However voriconazole-treated patients were more likely to perforate than natamycin-treated cases. Prajna et al. [17] stated that natamycin treatment was associated with significantly better clinical and microbiological outcomes than voriconazole treatment for smear-positive filamentous fungal keratitis, especially in patients with Fusarium infection. In this study we found that topical natamycin-treated Fusarium ulcers had fewer clinical scores, CFU, and less severe pathological findings than topical voriconazole-treated patients.

Other researchers reported that all of Fusarium species were sensitive to natamycin, but that did not translate to good clinical outcome in patients with Fusarium keratitis irrespective of early or late presentation. This probably indicates the poor penetration of natamycin especially in the presence of advanced fungal keratitis affecting deeper layers of the cornea [10]. Several studies have evaluated the effect of more potent drugs and delivery to the site of action in the posterior stroma using intrastromal or intracameral injections $[18,19]$.

Siatiri et al. [12] described the outcome in 3 patients with recalcitrant Fusarium keratitis and reported that intrastromal injection of voriconazole together with topical voriconazole is effective in reducing the infiltration size and control of the infection. Sharma et al. [20] offered intrastromal injection of voriconazole as a modality of treatment for managing cases of recalcitrant fungal keratitis. In this study we found that, in rabbits with Fusarium keratitis, intrastromal injection of voriconazole seems to be as effective as topical natamycin. It had lower clinical score, CFUs and fewer chronic inflammations, eosinophils, and anterior stromal vascularization than topical voriconazole. Since the cost of intrastromal injection of voriconazole is less than the frequent use of topical natamycin, it appears to be more economical to inject intrastromal voriconazole in Fusarium corneal ulcers. On the other hand, the use of one-time intrastromal injection of voriconazole is more comfortable than applying topical natamycin every hour. Moreover, intrastromal injection of voriconazole yields higher patient compliance. We believe that intrastromal voriconazole can be useful in the treatment of Fusarium keratitis especially in ulcers that do not respond to other treatment modalities. We propose further studies on different concentrations of intrastromal voriconazole to investigate their influence on Fusarium keratitis to find the most effective concentration.

\section{Disclosure}

This study was performed at Poostchi Eye Research Center, Poostchi Eye Clinic, Poostchi Street, Shiraz, Iran.

\section{Competing Interests}

The authors declare that they have no competing interests.

\section{References}

[1] S. Kotigadde, M. Ballal, Jyothirlatha, A. Kumar, R. Srinivasa, and P. G. Shivananda, "Mycotic keratitis: a study in coastal
Karnataka," Indian Journal of Ophthalmology, vol. 40, no. 1, pp. 31-33, 1992.

[2] N. Ando and K. Takatori, "Fungal flora of the conjunctival sac," American Journal of Ophthalmology, vol. 94, no. 1, pp. 67-74, 1982.

[3] R. B. Vajpayee, S. K. Gupta, U. Bareja, and K. Kishore, "Ocular atopy and mycotic keratitis," Annals of Ophthalmology, vol. 22, no. 10, pp. 369-372, 1990.

[4] M. J. Bharathi, R. Ramakrishnan, S. Vasu, R. Meenakshi, and R. Palaniappan, "Epidemiological characteristics and laboratory diagnosis of fungal keratitis. A three-year study," Indian Journal of Ophthalmology, vol. 51, no. 4, pp. 315-321, 2003.

[5] E. C. Poggio, R. J. Glynn, O. D. Schein et al., "The incidence of ulcerative keratitis among users of daily-wear and extendedwear soft contact lenses," The New England Journal of Medicine, vol. 321, no. 12, pp. 779-783, 1989.

[6] E. Speeleveld, B. Gordts, H. W. Van Landuyt, C. De Vroey, and C. Raes-Wuytack, "Susceptibility of clinical isolates of Fusarium to antifungal drugs," Mycoses, vol. 39, no. 1-2, pp. 37-40, 1996.

[7] I. Dóczi, T. Gyetvai, L. Kredics, and E. Nagy, "Involvement of Fusarium spp. in fungal keratitis," Clinical Microbiology and Infection, vol. 10, no. 9, pp. 773-776, 2004.

[8] G. Galperin, M. Berra, J. Tau, G. Boscaro, J. Zarate, and A. Berra, "Treatment of fungal keratitis from Fusarium infection by corneal cross-linking," Cornea, vol. 31, no. 2, pp. 176-180, 2012.

[9] P. A. Thomas, "Current perspectives on ophthalmic mycoses," Clinical Microbiology Reviews, vol. 16, no. 4, pp. 730-797, 2003.

[10] L. Pradhan, S. Sharma, S. Nalamada, S. K. Sahu, S. Das, and P. Garg, "Natamycin in the treatment of keratomycosis: correlation of treatment outcome and in vitro susceptibility of fungal isolates," Indian Journal of Ophthalmology, vol. 59, no. 6, pp. 512-514, 2011.

[11] W. E. Sponsel, J. R. Graybill, H. L. Nevarez, and D. Dang, "Ocular and systemic posaconazole (SCH-56592) treatment of invasive Fusarium solani keratitis and endophthalmitis," British Journal of Ophthalmology, vol. 86, no. 7, pp. 829-830, 2002.

[12] H. Siatiri, F. Daneshgar, N. Siatiri, and A. Khodabande, "The effects of intrastromal voriconazole injection and topical voriconazole in the treatment of recalcitrant Fusarium keratitis," Cornea, vol. 30, no. 8, pp. 872-875, 2011.

[13] T. G. Wu, V. V. Keasler, B. M. Mitchell, and K. R. Wilhelmus, "Immunosuppression affects the severity of experimental Fusarium solani keratitis," Journal of Infectious Diseases, vol. 190, no. 1, pp. 192-198, 2004.

[14] G. Alexandrakis, R. Haimovici, D. Miller, and E. C. Alfonso, "Corneal biopsy in the management of progressive microbial keratitis," American Journal of Ophthalmology, vol. 129, no. 5, pp. 571-576, 2000.

[15] H.-C. Lin, P.-H. Chu, Y.-H. Kuo, and S.-C. Shen, "Clinical experience in managing Fusarium solani keratitis," International Journal of Clinical Practice, vol. 59, no. 5, pp. 549-554, 2005.

[16] V. N. Prajna, P. S. Lalitha, J. Mascarenhas et al., "Natamycin and voriconazole in Fusarium and Aspergillus keratitis: subgroup analysis of a randomised controlled trial," British Journal of Ophthalmology, vol. 96, no. 11, pp. 1440-1441, 2012.

[17] N. V. Prajna, T. Krishnan, J. Mascarenhas et al., "The mycotic ulcer treatment trial: a randomized trial comparing natamycin vs voriconazole," The JAMA Ophthalmology, vol. 131, no. 4, pp. 422-429, 2013. 
[18] E. Garcia-Valenzuela and C. D. Song, "Intracorneal injection of amphothericin B for recurrent fungal keratitis and endophthalmitis," Archives of Ophthalmology, vol. 123, no. 12, pp. 17211723, 2005.

[19] K.-C. Yoon, I.-Y. Jeong, S.-K. Im, H.-J. Chae, and S.-Y. Yang, "Therapeutic effect of intracameral amphotericin B injection in the treatment of fungal keratitis," Cornea, vol. 26, no. 7, pp. 814818, 2007.

[20] N. Sharma, P. Agarwal, R. Sinha, J. S. Titiyal, T. Velpandian, and R. B. Vajpayee, "Evaluation of intrastromal voriconazole injection in recalcitrant deep fungal keratitis: case series," British Journal of Ophthalmology, vol. 95, no. 12, pp. 1735-1737, 2011. 


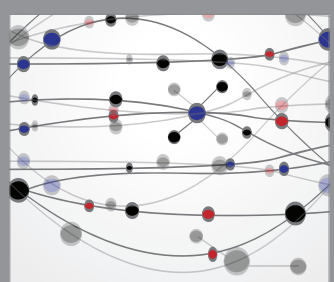

The Scientific World Journal
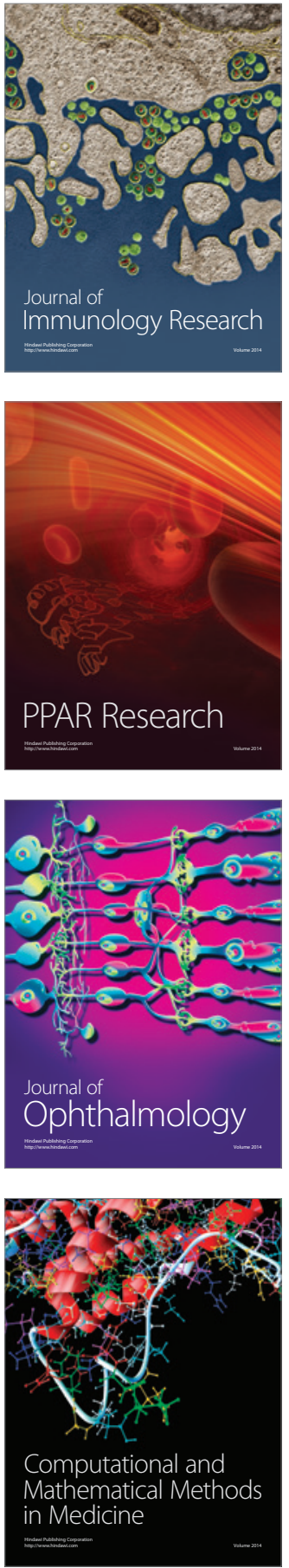

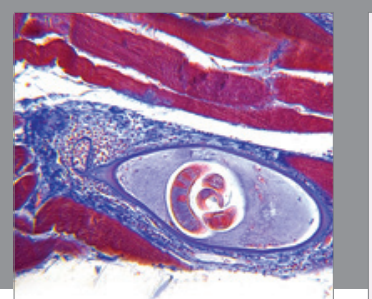

Gastroenterology Research and Practice

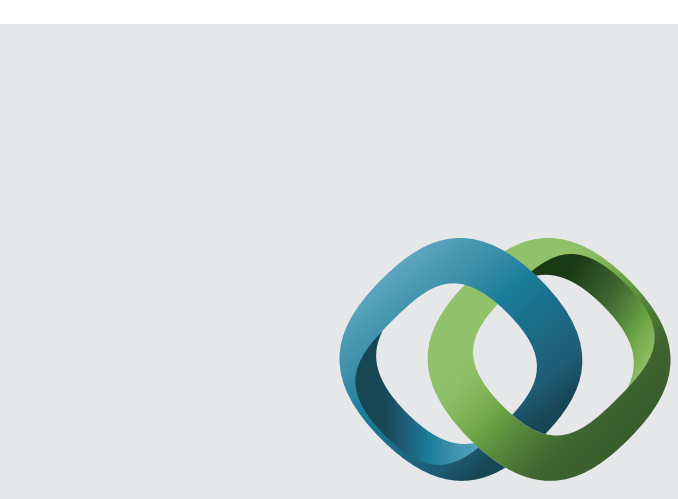

\section{Hindawi}

Submit your manuscripts at

http://www.hindawi.com
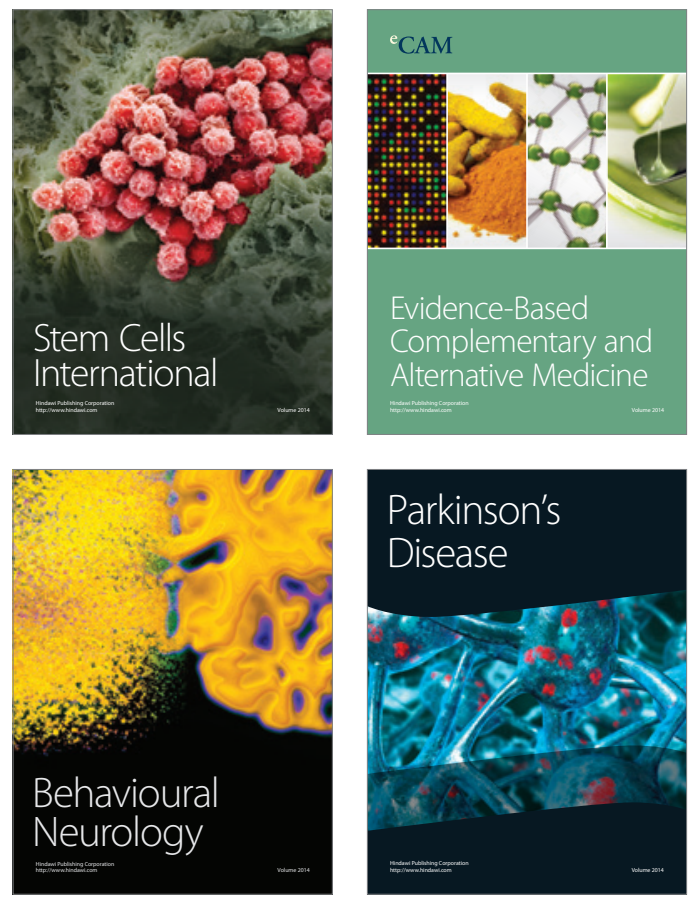
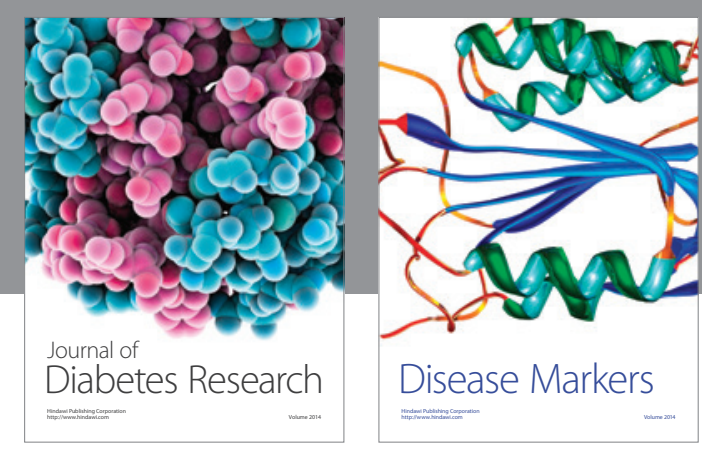

Disease Markers
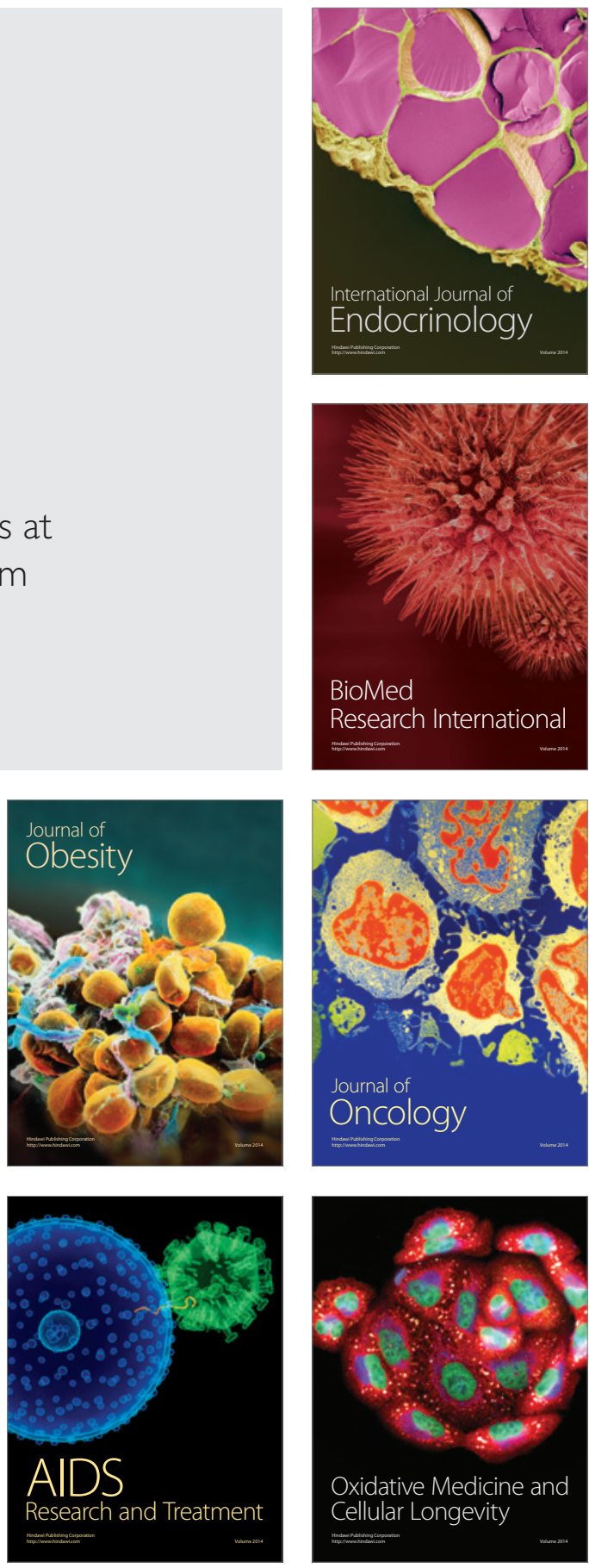